\title{
$\left.\mathbf{C}^{-}\right\rceil$CONGRESO
INTERNACIONAL \\ SOBRE \\ LF_O FOTOGRAFÍA
}

Congreso Internacional sobre Fotografía

UPV, 5 y 6 octubre 2017

Doi: http://dx.doi.org/10.4995/CIFo17.2017.6738

ISBN: 978-84-9048-604-7

\section{Los festivales de fotografía. El análisis cuantitativo como herramienta para matizar la historia de la fotografía.}

María Dolores Barrena Delgado

Doctora en Historia del Arte por la Universidad de La Laguna, Tenerife. madobade@gmail.com

\begin{abstract}
Ever since its dissemination, photography has earned a symbolic prestige and a space on its own. The photography festivals have contributed to this since the sixties of the 20th Century, and its study and assessment is one of the starting points for understanding its narrative. A narrative that has been built from premises that include the photographic image as a form of artistic communication, through the election of the representative and the movement of the images. Thus, conducting a research over the map of photographic encounters is also a way to formulate the history of photography.

This analysis has been developed considering 225 festivals from 70 countries, that were held between 1970, year of establishment of the Rencontres Internationales de la Photographie in Arlés, and 2015, date selected as the alleged year of economic recovery. For this research, social, economic and political changes as well as technological and artistic ones have been considered, and therefore the interpretation of its results is contextual from the point of view of space and time
\end{abstract}

Keywords: Photography, History of Photography, festival, dissemination, exhibition, statistics

\section{Resumen}

A partir de su difusión, la fotografía fue ganando en prestigio simbólico y en espacio propio. Los festivales de fotografia han contribuido a ello desde los años setenta del siglo XX, y su estudio y valoración suponen uno de los puntos de partida para entender un relato que se ha construido a partir de premisas que incluyen a la imagen fotográfica como comunicación artística, a través de la elección del representante y del movimiento de las imágenes. Investigar sobre el mapa de encuentros fotográficos es también una manera de formular la historia de la fotografía.

Este análisis se ha desarrollado a partir de 225 festivales de 70 países, celebrados entre 1970, año oficial de creación de los Rencontres Internationales de la Photographie de Arlés, y 2015, fecha elegida en tanto que se presupone el momento de recuperación económica. Para este 
Los festivales de fotografía. El análisis cuantitiavo como herramienta para matizar la historia de la fotografía Photography Festivals. The quantitative analysis as an instrument for qualifying the History of Photography.

estudio se han tenido en cuenta tanto los cambios sociales, económicos y políticos como los tecnológicos y los artísticos, por lo que la interpretación de los resultados es contextual desde el punto de vista del espacio y del tiempo.

Palabras clave: Fotografia, Historia de la Fotografia, festival, difusión, exposición, estadísticas.

\section{Introducción}

Las creaciones artísticas deben difundirse para ser conocidas e incluidas en la historia; una historia que desde lo cronológico, lo contextual, lo estético, lo categórico, lo formal, o cualquier otra opción va marcando pautas de percepción. La obra de arte, ya sea terminada, en forma de proceso o como eslabón de un discurso más amplio, culmina en su presentación. De esta manera, la difusión se convierte en cuestión fundamental en tanto que elección hacia lo público' ${ }^{1}$.

En los festivales, la difusión se lleva a cabo, en primera instancia, a través de la exposición y, es en la exposición donde se representan, a modo de escena, las obras que bajo una premisa predeterminada construyen el relato. El acto de representar significa, por un lado la interpretación, y, por otro, ser la imagen visible. Esa imagen visible, el representante, requiere ser designado, es decir, elegido. La unión de las partes implican un todo que se edifica mediante varios niveles de elección.

La evolución de todo medio de expresión y, en el caso de la fotografía, también de su tecnología asociada, genera a cada movimiento o innovación, una nueva avalancha de pensamiento que lleva aparejada cuestiones específicamente artísticas, discursivas y simbólicas, pero también otras tangenciales que se refieren a la industria, a la comunicación, al mercado, etc.

Por otra parte, los modelos de difusión han ido evolucionando y consolidándose como parte fundamental del sistema del arte. Así, desde las primeras relaciones de obras de los Salones parisinos a los formatos más avanzados de difusión a través de Internet, pasando por las revistas especializadas, catálogos, críticas e, incluso, artículos de promoción comercial, la fotografía se ha visto involucrada en distintos procesos de adaptación al medio elegido para su transmisión.

Los festivales son, desde 1970 — año de creación de los Rencontres Internationales de la Photographie de Arlés (Francia $)^{2}$ - , uno de los exponentes más importantes de divulgación de la fotografía tanto en su dimensión de imagen como en la de espacio reflexivo. En crecimiento desde la década de los setenta del siglo XX, aunque su formato no ha variado de manera sustancial su número sí lo ha hecho, y esta circunstancia requiere un estudio en

\footnotetext{
1 El contenido de esta comunicación es parte de la Tesis Doctoral inédita, El festival como modelo contemporáneo de disfusión de la fotografía. Guía internacional de festivales de fotografía, leída en enero de 2016, en la Universidad de La Laguna (Tenerife, España).

${ }^{2}$ Aunque en esa ciudad hubo un primer encuentro informal en el año 1969 organizado igualmente por los que serían los fundadores de los Rencontres, Lucien Clergue, Michel Tournier y Jean-Maurice Rouquette.
} 
profundidad que permite añadir algunos matices a la Historia de la Fotografía. Una gran cantidad de ciudades cuentan con un festival dedicado en exclusiva a la fotografía por lo que, además de funcionar como catalizadores de cuestiones económicas, turísticas y sociales — que forman parte de la mayoría de los análisis —, desde el punto de vista artístico son un contexto adecuado para la visualización de fotógrafos y fotógrafas, nuevas tendencias y movimientos, y para generar marcos de pensamiento.

Sus exposiciones sobre fotografía histórica han servido, por una parte, para validar la propia celebración del festival — considerándola un argumento de autoridad - y, por otra, para rescatar ciertas figuras que, debido a la reticencia de algunos sectores a considerar la fotografía como Arte, habían quedado en el olvido. Así, la actualización de la Historia de la Fotografía se ha ido construyendo en el transcurso de los últimos cuarenta años; incluso, el mercado del arte se ha visto influenciado por la entrada en escena de nuevas propuestas que, de no ser por los festivales, no dispondrían de una plataforma apropiada para la exhibición.

Investigar sobre el mapa de encuentros fotográficos, su frecuencia, número o crecimiento es también una manera de formular una historia transversal de la fotografía. Su cuantificación y análisis estadístico arroja nuevas perspectivas relacionadas con los principios básicos que subyacen en la definición de festival, en las formas de difundir la imagen y, sobre todo, pone en crisis algunas de las cuestiones que suelen asociarse a estas celebraciones a partir de lecturas superficiales, tales como, por ejemplo, las de centro y periferia o valoraciones extra artísticas.

\section{Objetivos}

El estudio de los festivales como uno de los modos de difusión más importantes supone pensar en términos de un evento en el que interactúan tanto la reflexión sobre la imagen fotogáfica como la exhibición y lugar de encuentro. Así, los objetivos de esta investigación son:

- Definir el concepto de festival y diferenciar los principios básicos del modelo.

- Conocer este modelo de difusión contemporáneo en términos cuantitativos.

- Desarrollar lecturas tangenciales igualmente necesarias para su análisis.

- A partir de los resultados, formular nuevas formas de entender este hecho cultural.

\section{Los festivales de fotografía. Definición, principios básicos, y cuantificación y estadísticas.}

El punto de partida para poder analizar de forma cuantitativa los festivales es definir el propio concepto e identificar los principios que subyacen en él. Así, el estudio de 225 festivales celebrados en setenta países en un

\footnotetext{
${ }^{3}$ Estudios amplios como el realizado por BenITo, L. (2001), Les festivals en France. París: L'Harmattan, en que se detallan los aspectos económicos; otros de carácter generalista como el de CASTELlOTE, A., «Los festivales de fotografia». En: Actas del Segundo Congreso de Historia de la Fotografia (2007). Zarautz: Photomuseum, pp. 21-27, en el que uno de los ejes de argumentación es el sector servicios y las respuestas econónicas; o los relacionados con la economía y la política como el de FonT-RÉAulX, D. (2010), «The Bold Innovations of a French Exhibition». En: Études photographiques. Francia: Societé Française de Photographie, número 25. https://etudesphotographiques.revues.org/3443 [Consulta: 14 de agosto de 2015; revisada 9 de septiembre de 2017], entre otros.
} 
Los festivales de fotografía. El análisis cuantitiavo como herramienta para matizar la historia de la fotografía Photography Festivals. The quantitative analysis as an instrument for qualifying the History of Photography.

arco temporal que abarca desde 1970 — año oficial de creación de los Rencontres Internationales de la Photographie de Arlés- y 2015 - fecha elegida en tanto que se presupone el momento de recuperación económica-, es una muestra lo suficientemente amplia como para llegar a descifrar estas cuestiones y conocer el modelo en profundidad.

El concepto actual de «festival» se crea en Francia, en 1947, a partir de la celebración del Festival de Aviñón ${ }^{4}$, aunque el término existía en el diccionario de la lengua francesa desde 1830 pero desde el punto de vista musical. La definición más aséptica es la referida a un evento con cierto grado de organización, que tiene una duración mínima en el tiempo, que se celebra de manera regular, donde se presentan trabajos nuevos e innovadores junto con obras de alta calidad y carga artística reconocida, para propiciar una mayor accesibilidad a la cultura por parte de la ciudadanía y crear nuevos públicos. Esta acepción no es suficiente para entender un fenómeno que tan rápidamente ha proliferado, y cuyos números crecen de forma exponencial. Por lo tanto, es necesario añadir que el festival implica, en teoría, la creación de programas especiales, diseñados con criterios no menos específicos, la aceleración y el afianzamiento de la creatividad local en un corto periodo de tiempo y la recepción de las distintas propuestas internacionales que generan intercambios y diálogos artísticos.

Los festivales derivan en bienales o trienales cuando la frecuencia de celebración deja de ser anual para repetirse cada dos o tres años, respectivamente. Desde el punto de vista conceptual, no hay diferencia por lo que utilizaremos «festival» como nombre genérico para referirnos a este formato de difusión con independencia de su periodicidad.

En el ámbito fotográfico, atendiendo a la cronología, la primera referencia en la época contemporánea es el «encuentro». Este término es utilizado en 1970 cuando Lucien Clergue, Michel Tournier y Jean-Maurice Rouquette organizan los primeros Rencontres Photographiques (Arlés, Francia) para reunir a fotógrafos y fotógrafas nacionales e internacionales; esta primera celebración partía, por analogía pero también por confrontación, de la tradición comenzada en el Festival de Aviñón.

De esta manera, el encuentro surge como espacio para el intercambio, la discusión y la visualización de la fotografía histórica y contemporánea, reunida en un solo lugar y durante unas fechas determinadas. Así, con los primeros Rencontres Photographiques en Arlés, se crea una estructura que ha perdurado en el tiempo. Si el objetivo era colocar a la fotografía en el lugar artístico que le correspondía, más allá de su consideración de documento, debían contemplarse distintas estrategias unidas en una misma acción: tenía que ser revulsiva y arriesgada, y tener la capacidad para generar el ambiente necesario de manera que toda la potencia creativa de la fotografía se diera cita.

\footnotetext{
${ }^{4}$ El Festival d'Avignon, fundado por Jean Vilar, se desarrolla a partir de una exposición de pintura y escultura contemporánea, al mismo tiempo que se expone la obra plástica, Vilar organiza la presentación de tres piezas escénicas de corte experimental. El objetivo fue captar un público joven al que mostrar un teatro contemporáneo alejado de la tradición.
} 
Este objetivo no podía conseguirse según los métodos que se habían venido desarrollando, ni tampoco en los lugares predestinados para el uso artístico. En este sentido, en un primer momento, a la exposición de obras de las grandes figuras de la fotografía internacional, como método de validación del medio, era necesario sumar la reflexión teórica; y, como modo de acercamiento a un público desconocedor que consideraba a la fotografía un mero artilugio de generación de imágenes, la vía de aproximación elegida fue el diálogo y la participación.

Desde entonces, esta estructura se ha mantenido, con sus matices y variaciones, pero, básicamente, los encuentros se definen en función de cuatro parámetros principales: la difusión de la fotografía a través de las exposiciones, la puesta en común de criterios y nuevos trazados de pensamiento, la confrontación de trabajos fotográficos recientes y la mezcla de públicos y participantes. Las derivaciones que han tomado estos cuatro aspectos son infinitas y, con el paso del tiempo, la estrategia ha sido, además de llevar la fotografía a las instituciones culturales, ocupar espacios no destinados a priori para la cultura. Esta última faceta es la que nos parece más interesante e influyente. Y, una vez más, son los primeros Rencontres los que marcan la pauta. En paralelo a la exposiciones ${ }^{5}$, se realizó una velada pública en el Ayuntamiento de Arlés que pretendía fomentar el pensamiento sobre el medio en una discusión abierta a todo el que quisiera participar. El hecho de efectuar esta sesión nocturna en el Ayuntamiento no fue casual. La «casa de todos» acogía así un acto cuyo fin fue colocar a la fotografía en un lugar hasta entonces no habitual.

\footnotetext{
${ }^{5}$ También se organizaron en el Museo Réattu de Arlés tres exposiciones cuya intención era, desde un primer momento y de manera clara y tajante, posicionar a la fotografía: la primera, «La photographie est un art»; otra con obras originales de Edward Weston, que había fallecido apenas diez años antes y cuya trayectoria era reconocida; y la tercera, una retrospectiva de Gjon Mili. Toda al información sobre los treinta primeros años de este festival puede encontrarse en GAUTRAND, JC. (1999). Avoir 30 ans. Croniques arlesiennes. Arlés: Acte Sud.
} 
Los festivales de fotografia. El análisis cuantitiavo como herramienta para matizar la historia de la fotografia Photography Festivals. The quantitative analysis as an instrument for qualifying the History of Photography.

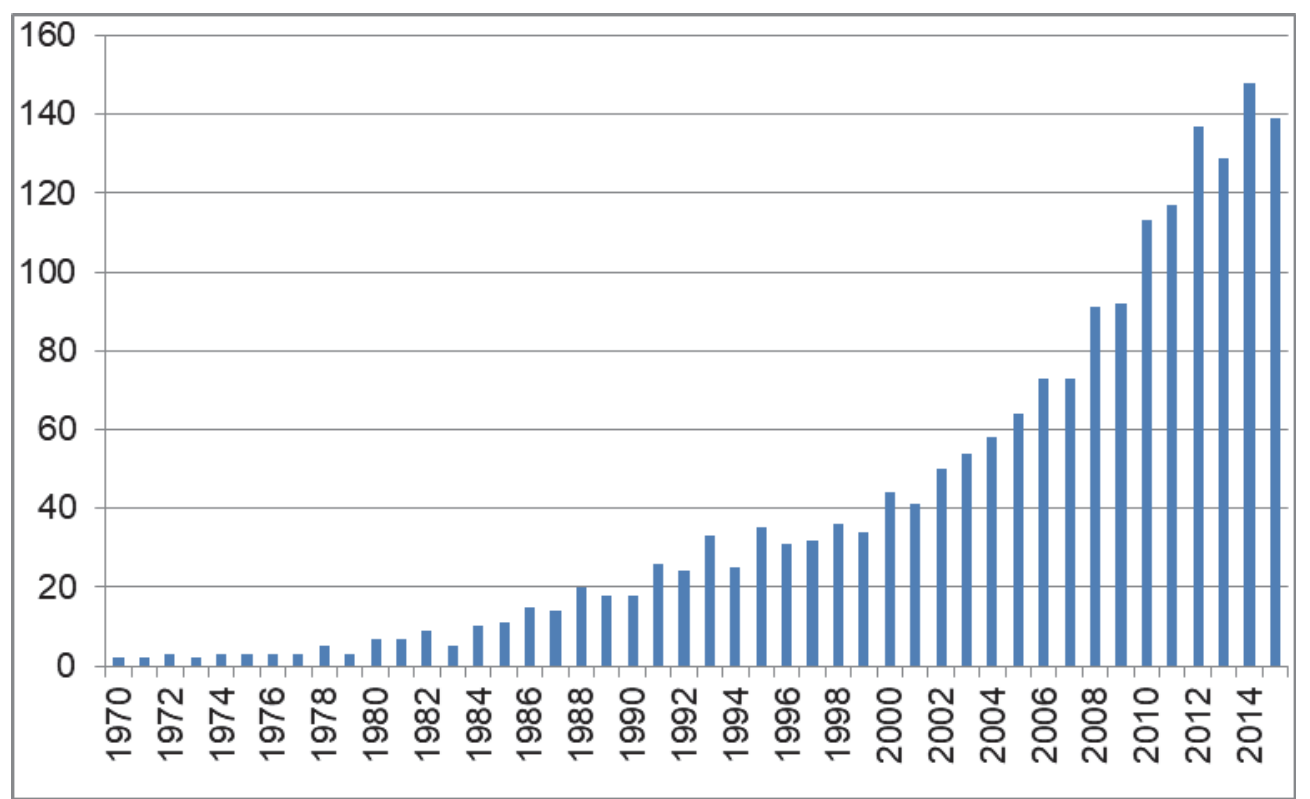

Fig. 1. Número de festivales por año.

En los años posteriores al inicio de los Rencontres de Arlés, comenzaron a aparecer algunas propuestas nuevas. De esta manera, cada año se celebraron entre uno y dos festivales hasta que en los primeros años de los ochenta se pasa a siete; once en 1985 y dieciocho en 1989 (Fig. 1). Este crecimiento coincide en origen con la organización del Mois de la Photo de París (1980) que será el modelo de otras celebraciones como, por ejemplo, Primavera Fotográfica ${ }^{6}$ (Barcelona, España, 1983). Por su parte, lo acontecido en Arlés, se desarrolla también en otras latitudes, como Houston, donde se constituye por primera vez, en 1986, el Fotofest, después de la visita de sus fundadores al encuentro arlesiano.

El modelo, según Anthony Georfieff — crítico de la revista European Photography_, prolifera pero con variaciones en su calidad. Para Georfiedd, el problema de muchos de estos festivales es que, frecuentemente, se centran en sí mismos y en el concepto de festejo, dejando a un lado la meta última que, en esos momentos, era la desmarginalización definitiva de la fotografía con respecto al resto de las artes ${ }^{7}$.

Indicio de esta referencia es que en el año 2015, la UNESCO Institute for Statistics publica un estudio realizado en 2009 sobre los festivales de todas las disciplinas que se celebran en la geografía mundial. La referencia más cercana en cuanto a la fotografía se traduce en una única expresión clave: Visual arts and crafts $^{8}$. Los criterios

\footnotetext{
6 VERdú I MARTí, M. (2003). 20 anys de Primavera Fotogràfica. Barcelona: Generalitat de Catalunya. http://cultura.gencat.cat/web/.content/sid/documents/arxiu/publicacions_estudis.htm_-_20anys_primfoto.pdf [Consulta: 27 de agosto de $2015 ;$ revisada 9 de septiembre 2017].

${ }^{7}$ GeORGIEFF, A. (1992). «56º Northern Latitud». En: European Photography. Berlín: European Photography (número 50, 1992, pp. 8-9).

${ }^{8}$ UNESCO Institute for Statistics (2009). «Festival Statistics: Key Concepts and Current Practice». En: 2009 UNESCO Framework for Cultural Statistics, Handbook. Bruselas: UNESCO, vol. 3, 2012. http://dx.doi.org/10.15220/978-92-9189-173-3-en [Consulta: 20 de septiembre de 2015; revisada 9 de septiembre de 2017]. Hemos preferido no traducir estos términos con el fin de evitar interpretaciones en su significado.
} 
para el desarrollo del estudio fueron sobre todo de tipo económico, y algunas reflexiones tangenciales concernientes a resultados sociales o medioambientales. Desde nuestro punto de vista, las razones para la creación de un festival han de ser, en primera instancia, culturales y, sus posibles consecuencias las enumeradas como necesarias por la Unesco.

Esta generalización resulta muy contradictoria teniendo en cuenta que de los 194 países existentes casi el 37\% celebra, o ha celebrado, uno o más festivales destinados en exclusiva a la fotografía, o a la fotografía y las artes visuales. Es decir, para ser más precisos: más de un tercio de los países geopolíticamente establecidos contiene o ha contenido en sus agendas culturales al menos un encuentro destinado a la fotografía y, en muchos casos, más de diez. La mayoría de estos festivales, muy por delante del resto de los continentes, se celebran en Europa (Tabla 1).

En cualquier caso, el mapa de festivales de fotografía celebrados entre el año 1970 y 2015 abarca la geografía mundial (Fig. 2), motivo suficiente para atisbar tanto su importancia como la necesidad de un estudio detallado, especialmente, si se tiene en cuenta la diversificación de la fotografía — desde la analógica hasta las democratizaciones últimas debidas a la accesibilidad de la imagen digital—, y su uso en términos de medio de expresión contemporáneo.

Tabla 1. Número de festivales por países (1970-2015)

\begin{tabular}{|c|c|c|c|c|c|}
\hline País & $\mathbf{N}^{\circ}$ Fest. & País & $\mathbf{N}^{\circ}$ Fest. & País & $\mathbf{N}^{0}$ Fest. \\
\hline Alemania & 12 & Finlandia & 5 & Nigeria & 1 \\
\hline Argentina & 2 & Francia & 28 & Noruega & 1 \\
\hline Australia & 7 & Gales & 2 & Nueva Zelanda & 1 \\
\hline Austria & 3 & Georgia & 1 & Países Bajos & 1 \\
\hline Bangladesh & 1 & Grecia & 5 & Panamá & 1 \\
\hline Bélgica & 2 & Guatemala & 2 & Perú & 1 \\
\hline Bosnia & 1 & Holanda & 6 & Polonia & 2 \\
\hline Brasil & 8 & Hungría & 1 & Portugal & 4 \\
\hline Bulgaria & 1 & India & 4 & Reino Unido & 2 \\
\hline Cabo Verde & 1 & Inglaterra & 4 & Rep. Dem. Congo & 1 \\
\hline Cambodia & 1 & Irlanda & 4 & Rep. Dominicana & 1 \\
\hline Canadá & 7 & Israel & 2 & Rumanía & 1 \\
\hline Chile & 1 & Italia & 10 & Rusia & 3 \\
\hline China & 9 & Japón & 3 & Serbia & 1 \\
\hline Colombia & 2 & Korea del Sur & 1 & Singapur & 1 \\
\hline Croacia & 2 & Letonia & 1 & Siria & 1 \\
\hline Dinamarca & 2 & Líbano & 1 & Sudáfrica & 1 \\
\hline Escocia & 1 & Lituania & 1 & Suecia & 2 \\
\hline
\end{tabular}


Los festivales de fotografia. El análisis cuantitiavo como herramienta para matizar la historia de la fotografia Photography Festivals. The quantitative analysis as an instrument for qualifying the History of Photography.

\begin{tabular}{|c|c|c|c|c|c|}
\hline Eslovaquia & 2 & Luxemburgo & 1 & Suiza & 3 \\
\hline Eslovenia & 1 & Malasia & 1 & Tailandia & 2 \\
\hline España & 28 & Mali & 1 & Turquía & 1 \\
\hline Estados Unidos & 19 & Marruecos & 1 & Uruguay & 1 \\
\hline Estonia & 1 & México & 3 & & \\
\hline Etiopía & 1 & Myanmar & 1 & & \\
\hline
\end{tabular}

Por lo tanto, la idea del festival se afianza, y su rápido crecimiento hizo que en el año 2000 se celebraran cuarenta y cuatro festivales y, lejos de agotarse, en 2006, se organizasen setenta y seis, es decir, casi el doble que apenas seis años antes (Fig. 1).

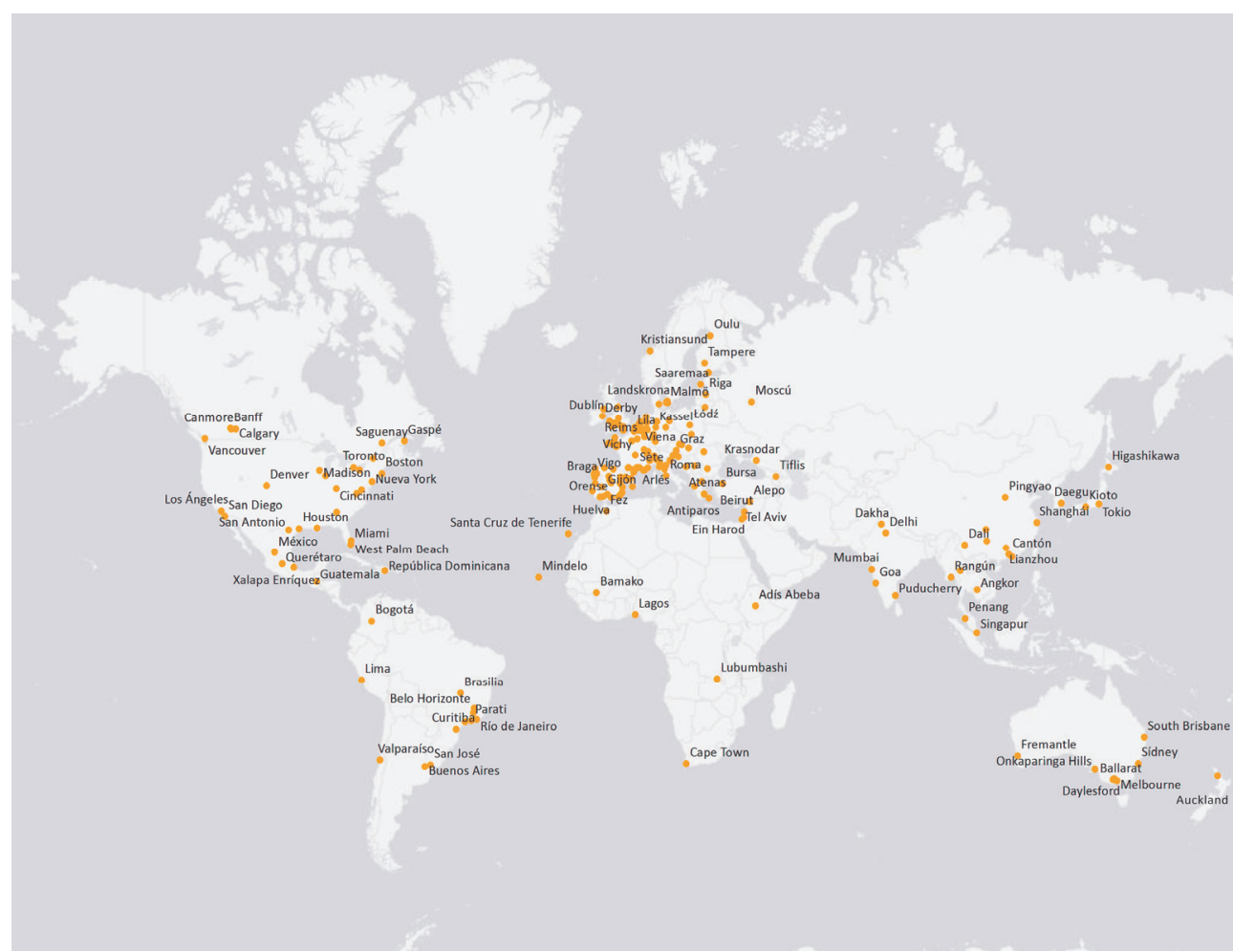

Fig. 2. Situación geográfica de festivales celebrados (1970-2015).

En este punto es necesario advertir que, a partir de 2007, se inician las políticas culturales de ayuda a la creación artística en Europa ${ }^{9}$; a ellas iban asociadas un programa de subvenciones entre 2007 y 2013 que, entre otras acciones, contemplaba la financiación parcial de festivales. El perfil de la gráfica referida a la geografía mundial (Fig. 3) es prácticamente igual a la que se dibuja cuando se aíslan los datos para el ámbito europeo. En este

\footnotetext{
9 «Culture Programe». En: European Comission. Education, Audiovisual and Culture Executive Agency. http://eacea.ec.europa.eu/culture/index_en.php [Consulta: 22 de agosto de 2015; revisada: 9 de septiembre de 2017]. De esta convocatoria solamente se beneficiarion los Rencontres de Arlés y los Encontros de Imagem de Lisboa en 2011 y Berlin Foto Festival. The Browse y Athens Photo Festival en 2013. Sin embargo, las ayudas fueron derivadas por otro tipo de subvenciones puesto que PhotoEspaña, durante varias ediciones contó con fondos europeos. Este último, en el catálogo de la edición de 2013, agradece especialmente a la Comunidad Europea, que es nuestro mayor apoyo, en Guía PhotoEspaña (2013). Madrid: La Fábrica, p. 17.
} 
sentido, este giro a favor de las propuestas culturales trajo consigo, efectivamente, la celebración de más festivales, de lo que pueden deducirse diversas posibilidades: la difusión de la fotografía y la reflexión sobre el medio aumenta, o el modelo puede llegar a banalizarse a través de festivales sin continuidad, contextos sin experiencia o simple excusa para otros fines, como había adelantado Gorfieff en 1992.

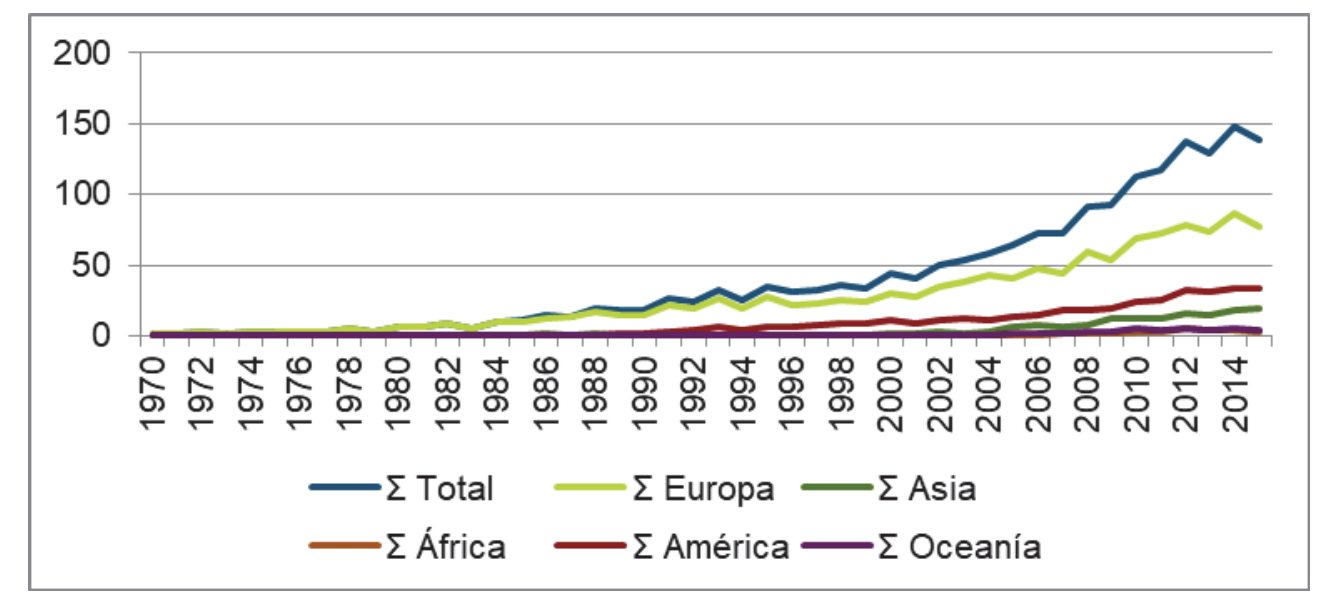

Fig. 3. Número de festivales por años y por continente.

En contraposición, en Estados Unidos la intervención en términos económicos de la administración pública está muy por debajo de la europea y la financiación proviene en su mayoría de agentes privados siendo, a priori, inferiores.

En 2010, el número aumenta un $300 \%$ con respecto a 2000, llegando a celebrarse hasta ciento trece. Entre los años 2012 y 2015 los datos se estabilizan con una media de ciento treinta por año, aproximadamente. Este dato llama la atención puesto que, en cronología, está ligado a la crisis financiera y al consecuente ajuste económico que supuso y que continúa vigente y, sin embargo, la celebración de festivales aumenta. Teniendo en cuenta sus costes y su corto periodo de duración esto puede deberse a varias pautas:

- El asentamiento definitivo de la fotografía como arte deviene en el interés por parte de las instituciones en este medio artístico.

- La necesaria inversión de capitales en el coleccionismo privado.

- La celebración de un festival aumenta nuevos valores culturales como las nuevas tendencias sobre «marca ciudad», cosmopolitismo, tejido artístico, etc. 
Los festivales de fotografia. El análisis cuantitiavo como herramienta para matizar la historia de la fotografia Photography Festivals. The quantitative analysis as an instrument for qualifying the History of Photography.

Ahora bien, ante esta diversificación y crecimiento de localizaciones, encontramos que, exceptuando los Rencontres de Arlés que es el iniciador del modelo y cuya trayectoria llega hasta nuestros días, la media de ediciones celebradas por festival es de cinco (Fig. 4); en algunos casos la cifra asciende a once y solo unos pocos superan las dos décadas de existencia. Este fenómeno, puede deberse al agotamiento del modelo en el contexto anfitrión, a la falta o la redistribución de los recursos económicos o, simplemente, a la interrupción de la propuesta.

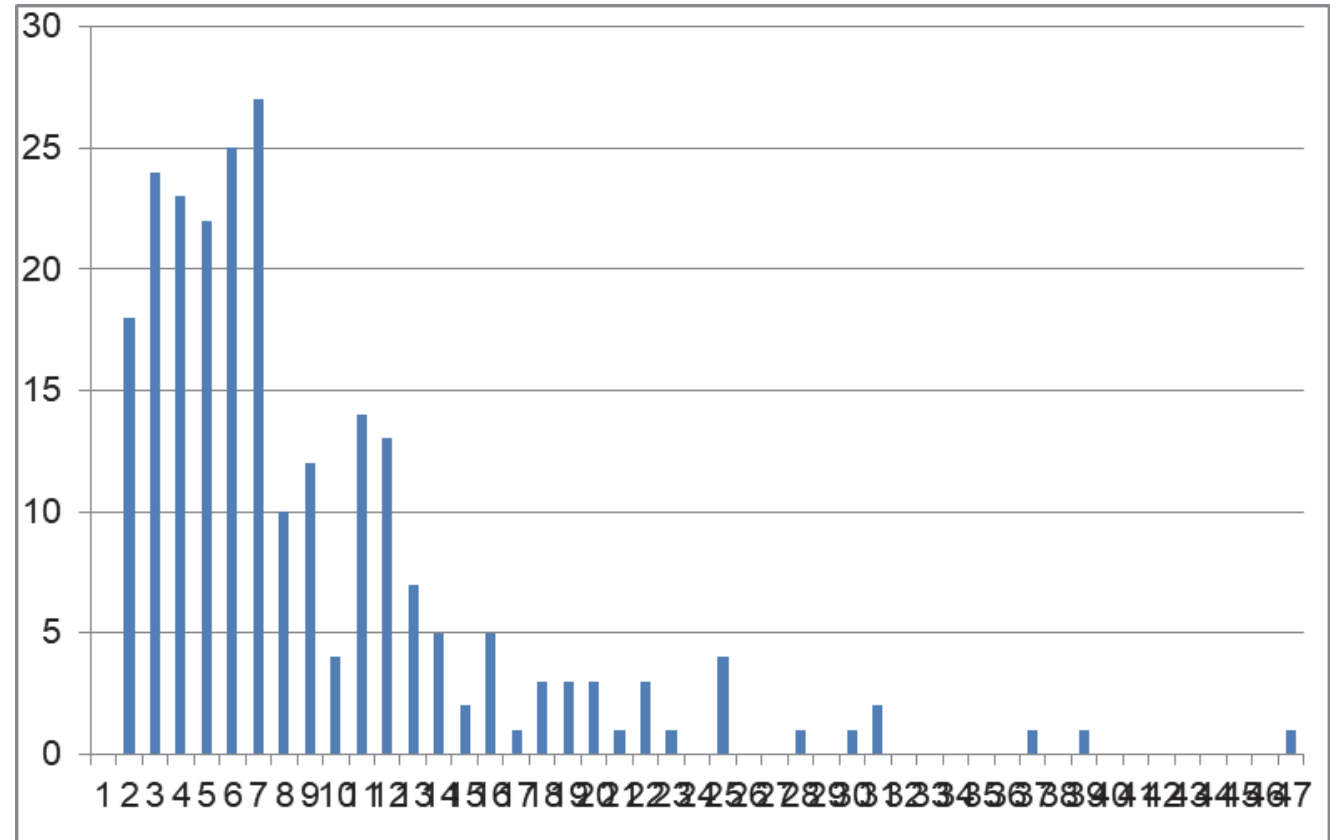

Fig. 4. Ediciones por festival (1970-2015).

Horizontal: $\mathrm{N}^{\mathrm{o}}$ ediciones alcanzadas.

Vertical: $\mathrm{N}^{\mathrm{o}}$ de festivales que alcanza ese número de ediciones.

Algunos autores se han manifestado sobre la cuestión cuantitativa. Dragan Klaic, presidente del Fórum Europeo para las Artes y el Patrimonio reflexionaba: La presión de los resultados y de las cifras se hace sentir y empuja a algunos a actuar de manera oportunista. [...] Raros son aquellos que se consagran como plataformas de innovación profesional, afirman sus opciones estéticas y nutren un debate y una reflexión críticos ${ }^{10}$.

En cualquier caso, parece obvio pensar que en una sociedad en la que el valor de la originalidad supone una de las bases de definición del sistema del arte, la organización continuada de un mismo acontecimiento signifique su suspensión si no ha conseguido alcanzar el estatus de hito — cada uno en su contexto_-, como sí ocurre con el Mois de la Photo de París, los Rencontres de Arlés, el Fotofest de Houston, la Internationale Photoszene de

\footnotetext{
${ }^{10}$ Citado por Pablo Berástegui en su texto de presentación de PhotoEspaña (2004) Madrid: La Fábrica, p. 8.
} 
Colonia o la Foto Biënnale de Rotterdam o, incluso, ejemplos algo menores ${ }^{11}$. Eso, unido al coste y al esfuerzo que supone un festival, cuya respuesta es la de un público restringido, hace que muchos hayan visto sus trayectorias truncadas o entrado en una espiral de innovación difícilmente sostenible.

Otra cuestión que muchos análisis han descrito para todas las estructuras artísticas es la de centro-periferia. En esta época de mundialización, las distancias no solo se acortan a través de Internet sino que la cultura ha experimentado una progresiva descentralización, proceso que comienza en los años setenta y que, en estos momentos, está en pleno apogeo. Así ocurre con las instituciones museísticas, las galerías, las ferias y todo tipo de elementos de difusión y distribución, y los festivales no podían quedar a un lado: la creación artística experimenta ese creciente jalonamiento geográfico que, poco a poco, convierte esas dos caras de la misma moneda en la esfera que alcanza cuando gira.

De esta manera, los conceptos de centro y periferia comienzan a estar diluidos y el formato festival se establece sin importar el contexto (Fig. 5). Desde pequeñas poblaciones (apenas mil habitantes) hasta los grandes centros neurálgicos acogen celebraciones que aúnan la obra tanto de reconocidos fotógrafos y fotógrafas como de los autores y autoras emergentes, la crítica más internacional y exposiciones que tratan temas de toda índole. Sin embargo, es importante destacar que la mayoría de los festivales tienen lugar en localidades cuya población oscila entre los cien mil y los quinientos mil y las ciudades entre uno y cinco millones de habitantes. Esto es, ciudades de pequeño y mediano tamaño. Este hecho se debe, probablemente a que el festival, además de ser un modelo contemporáneo de difusión de la fotografía es, sobre todo, una cuestión experiencial donde la condición de lugar de encuentro y de vivencia se superpone como capa sutil pero de fuerte importancia, en tanto que en la relación de unos trabajos con otros y de unos profesionales con otros, los festivales desarrollan toda su potencialidad; las cifras indican que es más abarcable en una ciudad de mediano tamaño que en en las grandes metrópolis. En cuanto a la cuestión económica, no todas las poblaciones disponen de las cuantías necesarias para abordar este tipo de eventos, tanto por su alto gasto como por las necesidades que colleva.

Así que si atendemos a los datos, podríamos concluir que la fotografía, desde los años setenta, es una cuestión de ciudades medianas más que de grandes centros, aunque no nos atrevemos a hacer esta afirmación tajante puesto que es muy problable que el rango de influencia simbólica entre unas y otros sea del todo desproporcionado

\footnotetext{
${ }^{11}$ Por ejemplo, Fotonoviembre. Bienal Internacional de Fotografia de Tenerife, cuya andadura comienza en esa Isla en 1991.
} 
Los festivales de fotografia. El análisis cuantitiavo como herramienta para matizar la historia de la fotografia Photography Festivals. The quantitative analysis as an instrument for qualifying the History of Photography.

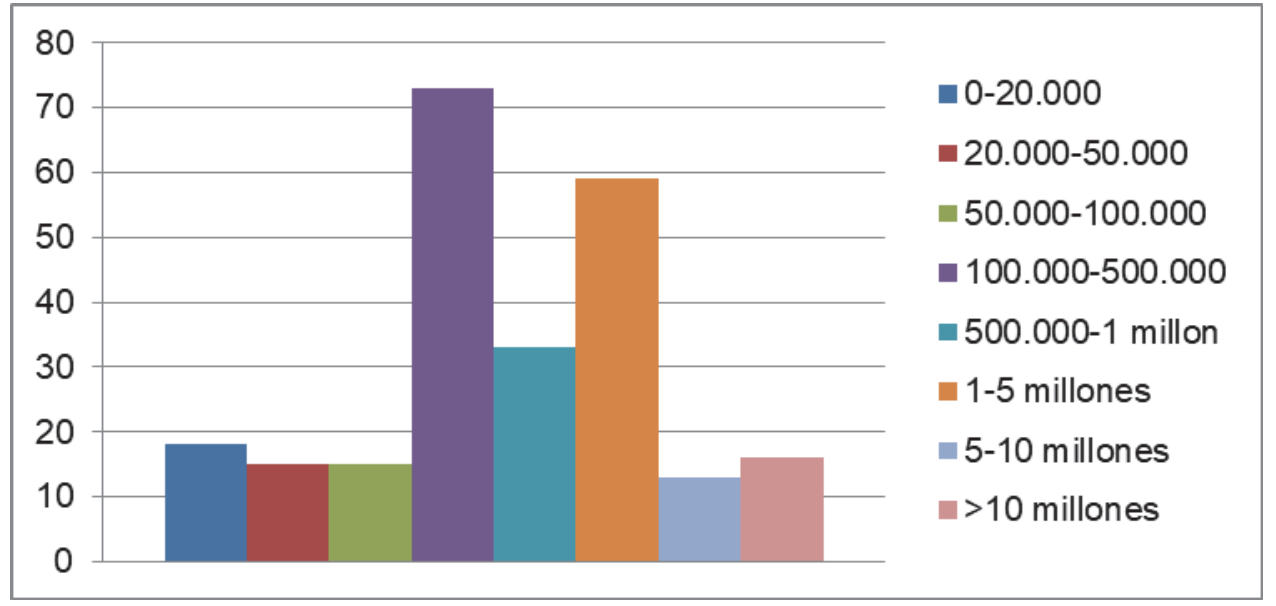

Fig. 5. Festivales por población (1970-2015).

\section{Sobre la difusión de la fotografía}

En cuanto a la difusion de la fotografía y, por lo tanto, de su entrada en los canales de conocimiento, parece lógico pensar que, a tenor de este análisis estadístico, los festivales generan una red con paradas, estaciones y caminos que se bifucan y multiplican, y que propicia el tránsito de propuestas de unos lugares a otros, de manera que las distintas ediciones de los configuran suponen un tejido que implusa el movimiento continuo de la fotografía en todas sus dimensiones. Además, la gran acogida que han tenido en la Modernidad estos encuentros hace suponer que, como quedaba recogido en el texto de presentación de la segunda edición, en 1991, de Le Mois de la Photo de Montreal, en unión con otras citas internacionales similares en París, Rotterdam, Barcelona o Houston, Montreal participa del tour ${ }^{12}$ como también lo harían más tarde otras como Madrid, Helsinki, Atenas, etc.; y, desde luego, la gran cantidad de exposiciones en las que derivó el crecimiento de lugares que destinaron un espacio y un tiempo a la fotografía ha hecho que, necesariamente, se abran otros temas de invesitgación y el númemo de trabajos se haya multiplicado.

Así, la Historia de la Fotografía, va incluyendo nuevos capítulos, por supuesto, sin renunciar a las figuras de reconocida trayectoria. En este sentido, esos «valores canónicos» se incluyen incansablemente en las exposiciones que forman parte de los festivales; en esta nómina se encuentran nombres como el ya citado Edward Weston y también Alfred Stieglitz, Eugene Atget, Henri Cartier-Bresson, Jacques Henri Lartigue, Guy Le Querrec, Robert Frank, André Kertesz, Robert Doisneau, Ralph Gibson, Manuel Álvarez Bravo, William Klein, Walker Evans, Diane Arbus, Man Ray, Irving Penn, Elliot Erwitt, Josef Koudelka, Josef Sudek, Jaroslav Rössler, Dorothea Lange, Paul Strand o Aaron Siskind, entre otros muchos; una segunda generación con una trayectoria consolidada que, en primera persona, forma parte de la gestación de los festivales, representada por

\footnotetext{
12 À jumeler aux événements internationaux parents de Paris, Rotterdam, Barcelone ou Houston, Montréal s'engage a son tour, avec ce deuxième Mois de la Photo à Montreal. Mois de la photo à Montreal (1991). Montreal: Vox Populi, p. 4.
} 
Duane Michals, Josef Koudelka, Graciela Iturbide, William Eggleston, Max Pam, Pedro Meyer, Bruce Davidson, Jan Saudek, Luis González de Palma, Miguel Rio Branco, Christine Spengler, Lee Freedlander, Franco Fontana, Robert Rauschenberg, Luigi Guirri, Harry Callahan, Andy Warhol o Joel Peter-Witkin; y, por último, los que son protagonistas de la evolución, metamorfosis y afianzamiento de la plena fotografía como arte y del peleno desrrollo del modelo de festival: Martin Parr, Joan Fontcuberta, Paul Graham, los representantes de la Escuela de Dusseldorf, Humberto Rivas, Cindy Sherman, Pierre Gonnord, Marcos López, Alberto García Alix, Isabel Muñoz, Cristina García Rodero, Nan Goldin, Flor Garduño, Mario Cravo Neto, Zang Huan, Yasumasa Morimura, Sebastião Salgado, Bernard Plossu, etc.

Por otra parte, acorde con las nuevas propuestas culturales y el pensamiento crítico general, con el pleno afianzamiento de la globalización y el surgimiento de voces manifiestamente discordantes o la asimilación de discursos que venían dándose en este sentido, a finales de los ochenta y los noventa, las programaciones comienzan a dar un giro hacia el concepto de identidad en todas sus dimensiones, que responde también a la necesidad de presentar nuevas propuestas que nutrieran la ingente cantidad de festivales a partir de los años noventa.

Por ejemplo, en 1990, la Fotografie Biënnale de Rotterdam (Holanda) centra su programación en la globalización, o mejor dicho, en el cuestionamiento de ésta. La portada de su catálogo supone todo un flash back: a través de la obra crítica de Mark Lewis, «We are the world / White noise», el autor unía el diseño del catálogo de «The Family of Man» (MoMA, 1955) con su trabajo en un arco temporal de casi cuarenta años. En análisis de esta elipsis, Bas Vroege, director del festival, argumentaba: La ideología que subyace en «The Family of Man», organizada por Steichen en 1955 [...] y la reducción de las culturas a una cuestión de moda o productos constityen la crítica de Lewis. [...] Su estrategia es comparable a la del montaje político anterior a la Guerra: luchar contra los medios utilizando sus propias estrategias. [...] Parece un buen método para preservar la identidad (cultural) en un mundo controlado por las corporaciones que están interesadas principalmente en crear mercados lo más grandes posibles para unificar productos, cualesquiera que sean éstos, maquinillas de afeitar, hamburguesas, publicidad, entretenimiento o reportajes periodísticos ${ }^{13}$.

Este giro hacia la identidad cultural en la elección del tema de esa edición de la Biënnale de Rotterdam, se completaba con la selección de una serie de países de interés: Unión Soviética, Canadá, Japón y Brasil. La justificación de esta presencia estaba relacionada por el desconocimiento de sus trayectorias culturales (Unión Soviética y Canadá), por su economía independiente (Brasil), y por la represión política o cultural que podían compartir todos ellos.

\footnotetext{
${ }^{13}$ The underlying ideology of Steichen's 1955 exhibition 'The Family of Man' and the reduction of other cultures to fashionable comodities are the subjects of Lewis's crititque. [...] His strategy is comparable to that of political montage from the pre-war period: to fight the media by using its own means. [...] It would seem to be an important suggestion, from the point of view too of preserving (cultural) identity in a world controlled by giant corporations that are mainly interested in creating as large a market as posible for their uniform products, whatever these may be, whether disposable razors, hamburgers, advertisements, amusement or journalistic reports. Fotografie Biënnale de Rotterdam (1990). Rotterdam: Uitgeverj 010 Publisher, p. 12.
} 
Los festivales de fotografía. El análisis cuantitiavo como herramienta para matizar la historia de la fotografia Photography Festivals. The quantitative analysis as an instrument for qualifying the History of Photography.

Un año antes, en 1989, el Mois de la Photo de Montreal surgía para poner en valor la fotografía canadiense y, especialmente, en primera instancia para darla a conocer. En el argumento de la tercera edición de 1993, las intenciones estaban claras: Esta tercera edición del MPM permitir centrar la atención del público, de los críticos $y$ de los organismos de difusión, en los artistas que tienen como punto en común la fotografia y un territorio geográfico heredado de una colonización muy reciente. Por lo tanto, estamos lejos de generar revelaciones de especificidad - ¿acaso un carácter distintivo? - de la fotografia de Quebec y canadiense. El tema elegido, 'ASPECTOS de la fotografia de Quebec y canadiense' proviene de un hecho constatado en los diferentes medios, aqui y en el extranjero: la fotografia de Quebec y canadientes no tiene -todavía-una difusión y un acogimiento proporcionales a su calidad ${ }^{14}$.

De esta manera, en su ámbito geográfico, cada festival se ocupa, obviamente, de las fotografías producidas por autores del lugar. Así ocurre también con los Rencontres de Bamako, dedicados casi en exclusiva a la difusión de la fotografía africana. Y las exposiciones incluidas en la programación apoyan estas nuevas derivas.

Con el título «At the borders: Photography and cultural identity», el Nordic Festival de Oulu (Finlandia) de 1991, puso de manifiesto las propuestas identitarias tomando como referencia los escritos de Roland Barthes, Susan Sontag o Walter Benjamin. Las fotografías trataron ese tema desde diferentes contextos y se planteaba la separación de la unidad con la que, hasta entonces, se percibía el norte de Europa ${ }^{15}$.

La reconfiguración de las líneas geopolíticas supuso también la fragmentación de un historia que no había cambiado en décadas y, por lo tanto, la consolidación de nuevas identidades o el despertar de otras. Lo que podríamos denominar un «resumen» de estos planteamientos ${ }^{16}$, es la exposición «Contemporary photography. Russia, China, Czech Republic \& Slovak Republics», que se organiza en el Fotofest 2014 y que fue comisariada por su directora Wendy Watriss. En ella, y bajo la premisa del régimen totalitario de estos países, se unen los trabajos de AES+F, Georgy Miofis, Pavel Banka, Gao Bo, Yao Lu, entre otros; el discurso en sí podía tener todo el sentido pero el título elegido parecía albergar intenciones que iban más allá de lo propuesto e, incluso, de la identidad. Esa edición del festival se completaba con una gran exhibición de fotografía árabe, también con la premisa del conflicto, y un conjunto de propuestas tejanas ${ }^{17}$.

\footnotetext{
${ }^{14}$ Cette troisième édition du MPM permettra d'attirer l'attention du public, des critiques et des diffuseurs sur le travail d'artistes ayant comme point comun la photographie et un territoire géographique hérité d'une colonisation sommes toute récente. Vous aurez donc compris que nous sommes bien loin d'une tentative de révélation des spécifités — du caractère distintict? - de la photographie québécoise et canadienne. Le theme choisi, 'ASPECTS de la photographie québécoise et canadienne, provient d'un constat que nous avons fait en cötoyant différents intervenants du millieu des arts, ici et à l'étranger: la photographie québécoise et canadienne ne connait pas —encore - une diffusion et un succès proportionnels à sa qualité. Mois de la Photo à Montreal (1993). Montreal: Vox Populi, p. 2.

${ }^{15}$ Tellgren, A. «At the borders». En: European Photography (1991). Berlín: Andreas Mulher-Polhe, número 48, 1991, pp. 13-15.

${ }^{16}$ A nuestro juicio, algo desatinados y carentes de estabilidad.

${ }^{17}$ Fotofest (2014). Houston: Fotofest, p. 190.
} 
Por lo tanto, en estos años, la identidad — nacional, regional, local— se vuelve primordial y se convierte en un nuevo campo de trabajo pleno de significados y opciones, a pesar de que estas cuestiones habían tenido manifestaciones en el pasado habían sido muy tímidas o habían pasado desapercibidas ${ }^{18}$.

Paralelamente, la creación fotográfica a partir del año 2000 se multiplica, o mejor dicho, se visualiza de manera superlativa de la misma manera que lo hacen los propios festivales. Ahora más que nunca y sin que haya una reflexión consciente de las necesidades contextuales, las citas se suceden y la vorágine de la difusión se acrecienta. Desde comisarios y comisarias omnipresentes ${ }^{19}$ a festivales con tintes de feria, las celebraciones se crean y se destruyen con la misma rapidez y, en algunos casos, no cuentan con una infraestructura lógica para su desarrollo. En otras ocasiones, se llevan a cabo en ciudades cuyo contexto es lo suficientemente fructífero en términos culturales como para que una propuesta de este tipo pueda llegar a consolidarse, dado que las dinámicas estables superan lo propuesto por el festival. Tal es el caso del New York Photo Festival ${ }^{20}$ o Photo London ${ }^{21}$. Este último celebra su primera edición en 2015. Casi con toda seguridad, puede afirmarse que más que a una cuestión de necesidad cultural, estas celebraciones están relacionadas con la mercadotecnia geográfica, es decir, colocar a la ciudad en el mapa y en el circuito.

Por otro lado, la introducción plena de la fotografía en las instituciones culturales - museos, fundaciones, colecciones públicas, etc.- , genera una demanda de obra que debe satisfacerse mediante adquisiciones. En cualquier caso, la difusión de la fotografía continúa y la diversificación de las propuestas inunda las salas de exposición sea cual sea la razón para la celebración de un festival. Aunque se heredan algunos intereses de décadas pasadas — identidad y puesta en valor de la fotografía de cada lugar-, la tendencia es a ampliar el campo de acción mediante las ideas de temas diversos o la inclusión, bajo el paraguas del festival, de un elenco de proyectos inconexos con el único fin de darles visibilidad. Por paradójico que parezca, se explica así que, en el marco de la crisis, los festivales de fotografía se hayan multiplicado en número a partir del año 2007 (ver Fig. 1). En términos de inversión, el creciente coleccionismo y la apertura de mercados propician nuevos espacios para la difusión.

\footnotetext{
${ }^{18}$ Por ejemplo, aunque en 1959 se celebró la Primera Exposición Latinoamericana de Fotografia en Sao Paulo [SougEZ, ML. (coord.) (2007). Historia general de la fotografia. Madrid: Cátedra, p. 532.], a excepción de algunas aportaciones que se dieron en torno a este tema en festivales o en las exposiciones dedicadas a autores de ese ámbito, hasta finales de los años ochenta no comenzó una verdadera reflexión. En 1986, el Mois de la photo de París dedicó varias muestras a autores de esas zonas. Así, tuvieron cabida desde la fotografía brasileña del siglo XIX hasta las manifestaciones más contemporáneas representadas por Marcos López, Juan Travnik o Facundo de Zuviria, o también clásicos como Manuel Álvarez Bravo; o las que fueron realizadas por integrantes de la agencia Magnum y que mostraban un «Monde nouveau», explorando el continente latinoamericano, su folclore tradicional y las tragedias de su historia.

${ }^{19}$ El formato deja de tener importancia en sí y se convierte en un lugar común para la difusión de la fotografía pero en términos superficiales. En cierto sentido, derivan en franquicias y los proyetos de los comisarios y comisarias —al margen de sus saberes y del interés de sus propuestas-, se convierten también en una suerte de lugar común, generando discursos que recorren las redes de difusión como en autopistas. Por ejemplo, Jean-Luc Monterosso, fundador y director del Mois de París, organiza también PhotoMed Liban en Beirut; Christian Caujolle, dirigió los Rencontres de Arlés, fue comisario invitado en PhotoEspaña, es el comisario de las tres últimas ediciones de Getxo Photo; Joan Fontcuberta, cofundador de Primavera Fotográfica, fue también comisario en los Rencontres de Arlés y, actualmente, en el Mois de la Photo de Montreal; Alejandro Castellote, fundador de FOCO Madrid, unos años más tarde fue comisario de PhotoEspaña en sus inicios, y ha participado en San José Photo y Daegu Photo, recientemente; Wendy Watriss y Frederic Baldwin dirigen el Fotofest desde sus inicios y lo mismo ocurre con Elda Harrington y los Encuentros Abierto de Argentina.

${ }^{20}$ Se trata de festival que, si bien cuenta con la estructura habitual del formato (exposiciones, discusiones y conferencias), tiene una duración de solamente cuatro días y sin aparente trascendencia.

${ }^{21}$ En teoría, pretende reunir en Londres a artistas, comisarios y público con el fin de pensar y ver fotografia a través, especialmente, de galerías que buscan visibilidad. Cuenta con un programa de conversaciones con artistas, performances, proyecciones y firmas de libros.
} 
Los festivales de fotografía. El análisis cuantitiavo como herramienta para matizar la historia de la fotografia Photography Festivals. The quantitative analysis as an instrument for qualifying the History of Photography.

\section{Conclusiones}

Los festivales han contribuido a la difusión de la fotografía y suponen un cambio de paradigma en cuanto a los modos de percepción de la creación fotográfica y de la propia consideración de la fotografía. Su conocimiento añade información sobre cómo se ha ido construyendo la Historia de la Fotografía y, sobre todo, cuáles son los motivos de su redacción. Proporcionan, en su conjunto, un estado actual de la creación fotográfica, entendida desde cualquier punto de vista, como corresponde a una disciplina que se establece como propia de la Modernidad.

Actualmente, la valoración del modelo desprende situaciones contradictorias. Por una parte, descansa sobre una estructura que cuenta ya con más de cuarenta años; por otra, está inserto en un sistema que ha cambiado con respecto a los años en los que éste inció su andadura. Los intereses entre 1970 y 2015 han mutado de manera sustancial pero la estrategia de desarrollo del festival sigue siendo la misma, de forma que esos cambios actuales se camuflan debajo de las programaciones.

De esta manera, la difusión se dispara en todas las direcciones y deriva por todos los caminos. Los motivos no son ya colocar a la fotografía en el estatus artístico sino ampliar los canales de visualización. Esto supone, aunque muy sutilmente, un cambio sustancial con respecto a los inicios. Como es lógico, en el ánimo de 1970 también existía la necesidad de abrir un nuevo mercado para la fotografía, sin embargo, en 2015, y dado el contexto económico, esa cuestión también ha crecido exponencialmente de la misma manera que lo ha hecho el número de festivales.

Desde sus inicios, un festival es sobre todo -0 debe entenderse de esta manera-, un hecho experiencial. Además de la presentación de una serie de exposiciones y eventos en torno al acto fotográfico, es el encuentro con artistas, críticos, comisarios, profesionales, coleccionistas, aficionados o público en general, lo que dota a este tipo de celebraciones del impulso necesario, facilitando no solo el acceso a los trabajos históricos sino también a los de reciente creación y, explican, en cierto sentido, lo que ha sucedido con la historia reciente, también en fotografía.

\section{Referencias}

BENITO, L. (2001). Les festivals en France. París: L'Harmattan.

BerÁstegui, P. (2004): PhotoEspaña. Madrid: La Fábrica, 2004.

Castellote, A. (2007). «Los festivales de fotografía». En: Actas del Segundo Congreso de Historia de la Fotografia. Zarautz: Photomuseum, pp. 21-27.

EACEA (2009). «Culture Programe». En: European Comission. Education, Audiovisual and Culture Executive Agency. Bruselas: EACEA.

http://eacea.ec.europa.eu/culture/index_en.php [Consulta: 22 de agosto de 2015; revisada 9 de septiembre de 2016]. 
Font-RÉAUlX, D. (2010). «The Bold Innovations of a French Exhibition». En: Études photographiques. Francia: Societé Française de Photographie, no 25, mayo 2010.

https://etudesphotographiques.revues.org/3443 [Consulta: 14 de agosto de 2015; revisada: 9 de septiembre de 2016]

Fotofest (2014). Houston: Fotofest.

Fotografie Biënnale de Rotterdam (1990). Rotterdam: Uitgeverj 010 Publisher.

GaUtrand, JC. (1999). Avoir 30 ans. Croniques arlesiennes. Arlés: Acte Sud.

GeORgIEFF, A. (1992). «56º Northern Latitud». En: European Photography. Berlín: Andreas Müller-Pohle, número 50, pp. 8-9.

Mois de la photo à Montreal (1991). Montreal: Vox Populi.

Mois de la Photo à Montreal (1993). Montreal: Vox Populi.

PhotoEspaña (2013). Madrid: La Fábrica.

SougEz, ML. (coord.) (2007). Historia general de la fotografia. Madrid: Cátedra.

Tellgren, A. (1991). «At the borders». En: European Photography. Berlín: Andreas Müller-Pohle, número 48, pp. 13-15.

UNESCO Institute for Statistics (2012). «Festival Statistics: Key Concepts and Current Practice». En: 2009 UNESCO Framework for Cultural Statistics, Handbook, vol. 3. http://dx.doi.org/10.15220/978-92-9189-173-3en [Consulta: 20 de septiembre de 2015; revisada 9 de septiembre de 2016].

Verdú i MARTí, M. (2003). 20 anys de Primavera Fotogràfica. Barcelona: Generalitat de Catalunya. http://cultura.gencat.cat/web/.content/sid/documents/arxiu/publicacions_estudis.htm___20anys_primfoto.pdf [Consulta: 27 de agosto de 2015; resvisada: 9 de septiembre de 2016]. 